\title{
Evaluación "in vitro" de la Actividad Antifungica del Aceite Esencial de Canela (Cinnamomum zeynalicum) Sobre Botrytis sp Aislado de Mora de Castilla (Rubus glaucus)
}

\author{
María Verónica González Cabrera, MsC \\ Vinicio Paredes Peralta, $\mathrm{MsC}$ \\ Fredy Erazo Rodríguez, $M s C$ \\ Tatiana Sánchez Herrera, MsC
}

Docente de Tiempo Completo de la Facultad de Ciencias Pecuarias Escuela Superior Politécnica de Chimborazo (ESPOCH), Ecuador

Juan Carlos Naranjo Herrera, $\mathrm{MsC}$

Docente de Tiempo Completo de la Facultad de Salud Pública

Escuela Superior Politécnica de Chimborazo (ESPOCH), Ecuador

Doi: 10.19044/esj.2019.v15n12p377 URL:http://dx.doi.org/10.19044/esj.2019.v15n12p377

\section{Resumen}

La canela es uno de los agentes naturales con poderes antimicrobianos más estudiados en los últimos años cuyo principal componente es el aldehído cinámico que posee actividad antibacterial, antifungica e inhibe la producción de micotoxinas. Por tal motivo esta investigación utiliza aceite esencial de Canela (Cinnamomum zeynalicum) como un potencial bioconservador frente a los microorganismos causantes de la pudrición de mora de castilla. Para ello se aisló al Botritys sp, como el principal factor microbiológico causante del deterioro en mora de castilla, luego de lo cual se realizó un procedimiento in vitro en medio de cultivo Papa Dextrosa Agar (PDA) en un diseño completamente al azar, con diferentes concentraciones de aceite esencial de canela en los que se demostró que los tratamientos con mayor actividad anti fúngica fueron a concentraciones de 250 y 500 ppm. Finalmente se evaluó la actividad anti fúngica del aceite esencial de canela in situ sobre fruta fresca a diferentes concentraciones, tiempo y temperatura, mediante el análisis de color, textura, sabor, olor, $\mathrm{pH}$, acidez y recuento de hongos y levaduras. Los experimentos in situ mostraron que el aceite esencial de canela a 500 ppm combinado con el almacenamiento de la fruta a temperatura de refrigeración $\left(5^{\circ} \mathrm{C}\right)$ fue el tratamiento más efectivo para reducir el deterioro y pérdida de la calidad de los frutos. 
Palabras clave: Aceite esencial de canela, Fungicida natural, Post cosecha, Mora de castilla

\title{
Evaluation "in vitro" of Anfungal Activity of Canela Essential Oil (Cinnamomum zeynalicum) About Botrytis sp Isolated from Castilla Blackberry (Rubus glaucus)
}

\author{
María Verónica González Cabrera, MsC \\ Vinicio Paredes Peralta, MsC \\ Fredy Erazo Rodríguez, MsC \\ Tatiana Sánchez Herrera, MsC
}

Docente de Tiempo Completo de la Facultad de Ciencias Pecuarias Escuela Superior Politécnica de Chimborazo (ESPOCH), Ecuador

Juan Carlos Naranjo Herrera, MsC

Docente de Tiempo Completo de la Facultad de Salud Pública Escuela Superior Politécnica de Chimborazo (ESPOCH), Ecuador

\begin{abstract}
Cinnamon is one of the most studied natural agents with antimicrobial powers in recent years whose main component is the cinnamic aldehyde that has antibacterial, antifungal activity and inhibits the production of mycotoxins. For this reason, this research uses Cinnamon essential oil (Cinnamomum zeynalicum) as a potential bioconservative against the microorganisms that cause castor mulberry rot. For this, Botritys sp was isolated, as the main microbiological factor causing the deterioration in arrears of Castilla, after which an in vitro procedure was carried out in Papa Dextrose Agar (PDA) culture medium in a completely randomized design, with different Concentrations of essential oil of cinnamon in which it was demonstrated that the treatments with greater antifungal activity were at concentrations of 250 and $500 \mathrm{ppm}$. Finally, the antifungal activity of the cinnamon essential oil in situ was evaluated on fresh fruit at different concentrations, time and temperature, through the analysis of color, texture, taste, smell, $\mathrm{pH}$, acidity and count of fungi and yeasts. The in situ experiments showed that the essential oil of cinnamon at $500 \mathrm{ppm}$ combined with the
\end{abstract}


storage of the fruit at refrigeration temperature $\left(5^{\circ} \mathrm{C}\right)$ was the most effective treatment to reduce the deterioration and loss of the quality of the fruits.

Keywords: Cinnamon essential oil, Natural fungicide, Post-harvest, Castilla Blackberry

\section{Introduction}

La mora de castilla (Rubus glaucus B.) es un fruto no climatérico (INEN, 2010) altamente perecedera por su gran contenido de agua, frágil a la manipulación, susceptible al ataque de hongos y que presenta elevadas pérdidas post cosecha (Ramírez, 2012; Ayala et al., 2013; Guzmán et al., 2018). En la etapa de almacenamiento, se produce un ablandamiento progresivo de la pulpa que ocurre con mayor intensidad durante los primeros días después del cosechado. Esta disminución pérdida de firmeza, es el principal limitante del tiempo de conservación. Consecuentemente su vida de anaquel es corta (3-5 días a 0-1 ${ }^{\circ} \mathrm{C}$ y 80-95 \% HR), registrándose pérdidas pos cosecha entre 70 - 80\% (Ramírez, 2012; Ayala et al., 2013; Villegas et al., 2016). En los sitios de venta donde las temperaturas de refrigeración son superiores a los $5^{\circ} \mathrm{C}$ la senescencia del fruto se acelera, acortando su vida útil a 1-2 días, y haciendo visibles numerosos cambios como: reblandecimiento, elevada coloración, deshidratación, contaminación por mohos etc. (Kaume et al., 2012). Estos cambios post cosecha están asociados a alteraciones bioquímicas que conducen a la maduración del fruto y su manifestación depende de diversos factores como: temperatura de almacenamiento, carga microbiana, daños del fruto entre otros (Villegas et al., 2016).

Estos efectos negativos del almacenamiento vienen acompañados de manera general por la aparición de la pudrición consecuencia principalmente de la presencia de microorganismos como Erwina, Pseudomonas, Penicillium, Botrytis, Aspergillus y Fussariun (Reina, C., 1998) Está pérdida de atributos de calidad de la fruta post cosecha influye de manera negativa a nivel comercial y económico.

Según la $8^{\text {a }}$. Edición de la farmacopea francesa (1965), los aceites esenciales son "productos de composición general muy complejas que contienen los principios volátiles que se encuentran en los vegetales más o menos modificados durante su preparación" Sánchez et al., (2008), define a los aceites esenciales como mezclas químicas de metabolitos secundarios sintetizados en determinadas partes de las plantas (hojas, flores y/o raíces), que le atribuyen un aroma característico. Estas mezclas químicas complejas incluyen la presencia de terpenos junto con otros compuestos casi siempre oxigenados como alcoholes, éteres, ésteres, aldehídos y compuestos fenólicos. En general, la función biológica de los terpenoides de los aceites 
esenciales sigue estando poco clara. Sin embargo, es probable que tengan un papel ecológico (Saltos A., 2001).

Por todas estas propiedades, se ha evaluado la actividad que pueden ejercer estos compuestos contra microorganismos patógenos en alimentos, y por tanto, la posibilidad de ser utilizados como un método de eliminación. Raybaudi, Massilia, Soliva y Martín (2016) consideran que existe una tendencia reciente con respecto al uso y aplicación de aceites esenciales como medios de control frente al desarrollo de microorganismos patógenos, gracias a sus características antimicrobianas que les confiere un gran potencial en la conservación de alimentos. Según la FAO (2006), el aceite de hoja de canela (Cinnamomum zeylanicum) contiene como componente principal 75-85\% de eugenol, con una alta actividad antibacterial, y contiene $5 \%$ de cinamaldehído, el cual contribuye con su carácter aromático y características antimicrobianas.

Dentro de los trabajos previos realizados en el campo de la microbiología de alimentos, destacan investigaciones que incluyen a aceites de clavo (Syzygium aromaticum), canela (Cinnamomum zeylanicum) y tomillo (Thymus vulgaris); entre otros como agentes de inhibición para el crecimiento micelial y la esporulación de los conidios de Colletotrichum (C. gloeosporioides), a concentraciones de 200, 250 y $300 \mu \mathrm{g} \mathrm{mL-1} \mathrm{(Tsao,} \mathrm{Zhou,}$ 2000).

De la misma manera, se observó un efecto inhibitorio en la investigación realizada por (Barrera, Garduno, \& García, 2009), denominada "Actividad antifúngica in vitro de aceites y sus compuestos en el crecimiento micelial de Fusarium oxysporum f. sp. gladiolos (Massey, Snyder y Hansen)", con los mismos aceites esenciales mencionados en el párrafo anterior y bajo las mismas condiciones. Asimismo García, E. et al. (2006), evaluaron el efecto del aceite esencial de canela sobre A. flavus utilizando una dosis mínima de 100 ppm y una máxima de 500 ppm. Por su parte, Plotto et al. (2003), determinaron el efecto de varios aceites esenciales, incluyendo tomillo y canela para el control de enfermedades en tomate y observaron que el aceite esencial de canela fue uno de los mejores para el control de B. cinérea.

En este sentido la presente investigación tiene como propósito realizar una evaluación "in vitro" de la actividad antifungica del aceite esencial de canela (Cinnamomum zeynalicum) sobre Botrytis sp aislado de mora de castilla (Rubus glaucus).

\section{Metodología}

La investigación tuvo lugar en el laboratorio de Bioquímica y Alimentos de la Facultad de Ciencias de la Escuela Superior Politécnica de Chimborazo, laboratorio de la división de Aguas y Alimentos LIBEXLAB en 
la ciudad de Quito; así como en los laboratorios dela empresa ISABRUBOTANIK S.A. ciudad de Ambato provincia del Tungurahua.

En el material vegetal se utilizaron frutas de mora de castilla provenientes de cultivos comerciales ubicados en el sector Huachi Grande, cantón Ambato, provincia de Tungurahua. Para la selección de las frutas se realizó un muestreo no probabilístico y se consideraron aspectos como grado de madurez, ausencia de enfermedades criptogámicas, ausencia de lesiones físicas y mecánicas, sanas y limpias, de acuerdo a lo especificado por el Instituto Colombiano de Normas Técnicas NTC 4106 (ICONTEC, 1997).

El aceite esencial de Canela fue suministrado por la empresa ISABRUBOTANIK S.A, obtenido con el método de extracción con solventes de corteza y hojas del árbol de Cinnamomun zeynalicum en su planta de producción.

\section{Caracterización físico - químico de las frutas}

Se evaluaron parámetros de longitud, diámetros, volumen, peso, empleando 25 frutos por muestra y grado de madurez analizado, según NTC 4106. La medición de longitud, diámetro mayor y menor se realizó con empleo de un calibrador HOPEX, el peso se determinó en balanza de precisión electrónica HR-200, con capacidad de 210 g $\pm 0,0001$ g. El contenido de sólidos solubles, pH y Acidez titulable se determinó según metodologías reportadas por A.O.A.C. Official Methods of Analysis (2000), 942.15 (B) (37.1.37). La medición del pH se realizó con potenciómetro marca SCHOTT modelo CG 820 a temperatura de referencia de $20^{\circ} \mathrm{C}$ y la acidez titulable se reportó en porcentaje de ácido málico.

\section{Análisis Microbiológico}

- Recuento de hongos (mohos y levaduras): Para este ensayo se utilizó la NTE INEN 1529-10, Recuento en placa por siembra en profundidad, Utilizando una sola pipeta estéril, pipetear por duplicado alícuotas de $1 \mathrm{~mL}$ de cada una de las disoluciones decimales en la placa petri adecuadamente identificadas. El medio de cultivo escogido fue Saboraud Dextrosa fundido y templado a $45 \pm 2{ }^{\circ} \mathrm{C}$. Las placas se incubaron a una temperatura que osciló entre 22 y $25^{\circ} \mathrm{C}$ por 5 días; examinando a los 2 días y comprobar si se ha formado o no micelio aéreo.

- Identificación de hongos (mohos y levaduras): La determinación de los hongos causantes de la pudrición en mora fresca se realizó por el método de observación directa al microscopio de una pequeña muestra de las frutas infestadas con hongos, comparando posteriormente con las características morfológicas señaladas en bibliografía. Se dejó a temperatura ambiente las frutas por cinco días hasta cuando empieza 
la pudrición causadas por diversos hongos. Se tomó una muestra pequeña de hongos cultivados y se colocaron en el cubre objetos, adicionando lacto fenol hasta cubrir completamente la muestra de hongos cultivados. Se observó al microscopio y se dibujó las estructuras de los hongos encontrados para luego comparar con la bibliografía consultada (Ellis, 1971).

- Aislamiento de Botrytis sp: Consistió en la obtención de un cepa pura del hongo aislado de la muestra de hongos cultivados. Con el sacabocados de $4 \mathrm{~mm}$ de diámetro se toma una muestra que fue inoculada en cajas petri con Potato Dextrose Agar (PDA). Este procedimiento se repite hasta obtener cepas de Botrytis sp puras.

- Elaboración de los medios de cultivo: El medio de cultivo, Papa Dextrosa Agar (PDA) se reconstituyó con agua de acuerdo a las condiciones indicadas por el fabricante (Bioxon). Fueron utilizados 50 mg del Aceite esencial de Canela disueltos en $500 \mu \mathrm{L}$ de Tween 20. La concentración final de esta disolución fue 100000 ppm. Se realizaron diluciones al décimo, utilizando tubos de ensayo, secos, limpios y estériles a los que se añadió $900 \mu \mathrm{L}$ de Tween 20 y $100 \mu \mathrm{L}$ del extracto concentración 100000 ppm, las concentraciones finales fueron de $10000 \mathrm{ppm}$ y $1000 \mathrm{ppm}$.

Se prepararon varias diluciones sucesivas hasta concentraciones finales de: 500, 250 y $125 \mathrm{ppm}$. Se codificaron cajas Petri estériles con la concentración final y se procedió a pipetear separadamente $100 \mu \mathrm{L}$ de las disoluciones con el aceite esencial de canela a los tubos de ensayo que contenían $10 \mathrm{~mL}$ de PDA a $45^{\circ} \mathrm{C}$. Se procede a homogeneizar con la ayuda de un vórtex y al llenado de las cajas petri. Una vez que se solidificó el medio de cultivo, se sometieron a prueba de esterilidad, la cual consiste en dejarlos en incubación a $25^{\circ} \mathrm{C}$ durante 24 horas. García, E. et al. (2006).

\section{Evaluación antifungica in vitro del aceite esencial de canela}

Se evaluaron cuatro diferentes concentraciones de aceite esencial de canela: $125 \mathrm{ppm}, 250 \mathrm{ppm}, 500 \mathrm{ppm}$ y $1000 \mathrm{ppm}$, las cuales presentan antecedentes de efecto antifúngico García, E. et al. (2006). (Ver Tabla 1). Los aceites esenciales se incorporaron al medio de cultivo PDA como se describió anteriormente, después de la prueba de esterilidad se sembró un inóculo de Botrytis a manera de explantes discoidales de $4 \mathrm{~mm}$ de diámetro tomados con un sacabocado de una colonia de siete días de desarrollo, incubada a $25^{\circ} \mathrm{C}$ y se colocaron en el centro de las cajas de Petri con los tratamientos. El experimento se incubó a $25^{\circ} \mathrm{C}$ durante once días. Se realizaron tres repeticiones por cada tratamiento. Las cajas Petri con PDA fueron utilizadas 
como control positivo y las cajas con PDA más dicloran a 1 ppm representaron al control negativo.

Posteriormente se evaluó el desarrollo de las colonias, considerando un efecto fungicida en aquéllos que no presentaron crecimiento del hongo y fungistático para los que sí lo presentaron. Finalmente se eligieron solo dos concentraciones; 250ppm y 500 ppm, que se utilizaron en los ensayos posteriores. Las cajas con PDA más Tween 20, se utilizaron como control para descartar que el Tween 20 tenga efecto sobre el hongo. Se hicieron 3 repeticiones por experimento. Los medios inoculados se incubaron a temperatura ambiente $\left(25-28^{\circ} \mathrm{C}\right)$, registrando cada $24 \mathrm{~h}$ la lectura del diámetro de la colonia, hasta que el control positivo cubrió por completo la superficie de la caja.

\section{Análisis estadístico}

En todos los experimentos se utilizó un diseño experimental completamente al azar en arreglo simple. En los ensayos in vitro los datos obtenidos de crecimiento, micelial y esporulación se analizaron de acuerdo a un ANOVA de una vía y la comparación de medias se hizo con la prueba de Tuckey, cabe destacar que se analizaron todos los datos diferentes de cero; utilizando el programa SPSS STATISTICS versión 19.0.

Los datos que se reportan fueron obtenidos sobre la base de la metodología indicada.

1. Variables medidas en el almacenamiento de la mora de castilla (Rubus glaucus) bajo dos condiciones.

Una vez en el laboratorio, se caracterizaron las frutas cosechadas y se almacenó a las dos condiciones propuestas, ambiente $\left(\mathrm{T}=21^{\circ} \mathrm{C}\right.$ y $\mathrm{HR}$ de $67 \%$ ) y refrigerada $\left(\mathrm{T}=5^{\circ} \mathrm{C}\right.$ y HR $\left.90 \%\right)$, se obtuvieron los resultados presentados en la Tabla $\mathrm{N}^{\mathrm{o}} 1$ donde se evaluaron las siguientes variables: Características sensoriales (Color, Textura, Olor y Sabor), pH, Acidez titulable, y recuento de Mohos y Levaduras para ambas condiciones. 
Tabla 1. Descripción de los tratamientos utilizados para la determinación de las propiedades antifúngicas del aceite esencial de canela (Cinnamomum zeylanicum) sobre Botrytis sp.

\begin{tabular}{|c|c|c|c|}
\hline MUESTRAS & $\begin{array}{l}\text { CONCENTRACIÓN } \\
\text { DEL ACEITE } \\
\text { ESENCIAL }(\mathbf{p p m})\end{array}$ & CÓDIGO & $\begin{array}{l}\text { TIEMPO DE } \\
\text { OBSERVACIÓN EN } \\
\text { DÍAS }\end{array}$ \\
\hline M1 & PDA & Control & $3-5-7-9-11$ \\
M2 & PDA - Tween 201ppm & PDT & $3-5-7-9-11$ \\
M3 & 125 & $\mathrm{CA}_{1}$ & $3-5-7-9-11$ \\
M4 & 250 & $\mathrm{CA}_{2}$ & $3-5-7-9-11$ \\
M5 & 500 & $\mathrm{CA}_{3}$ & $3-5-7-9-11$ \\
M6 & 1000 & $\mathrm{CA}_{4}$ & $3-5-7-9-11$ \\
M7 & 2000 & $\mathrm{CA}_{5}$ & $3-5-7-9-11$ \\
M8 & D1 & $3-5-7-9-11$ \\
\hline
\end{tabular}

En los procedimientos realizados a $21^{\circ} \mathrm{C}$ de almacenamiento en Mora de Castilla, estas frutas presentaron síntomas de Botrytis, al día 5, lo cual causó daños en aproximadamente el $12 \%$ de la fruta muestral, y se incrementó en días posteriores. En el día 7, aproximadamente el $92 \%$ de la fruta estuvo infectada de Botrytis, dejándola inservible. A $5{ }^{\circ} \mathrm{C}$, el $20 \%$ de la fruta muestral almacenada fue atacada por hongos, con incrementos en días posteriores. Hasta el día 5 no hubo presencia de hongos, pero a partir día 7 se evidenciaron y a estas condiciones el $60 \%$ de la fruta era inservible.

Los cambios químicos como el $\mathrm{pH}$, en los días postcosecha presentaron en las dos condiciones de almacenamiento una tendencia ascendente, mientras que la acidez titulable descendió durante estos mismos días. Como característica especial se puede observar que en los tres días de almacenamiento al medio ambiente la fruta presentó una disminución en la acidez titulable de un valor de 3,2 a 2,59 expresado como porcentaje de ácido málico.

En los ensayos realizados se pudieron establecer dos condiciones de almacenamiento a $21^{\circ} \mathrm{C}$ y a temperatura de refrigeración $5^{\circ} \mathrm{C}$. Como se puede observar en la Figura 1 la acidez titulable presenta porcentajes que van decreciendo para ambas condiciones de almacenamiento. Se puede determinar de igual manera que en condiciones de refrigeración los cambios se producen más lentamente, la acidez titulable de la fruta bajo refrigeración, disminuye de un valor de 3,2\% el día de la recolección a un 2,20\% en el séptimo día del almacenamiento (expresada en porcentaje de ácido málico). En lo referente a la segunda condición de almacenamiento $\left(21^{\circ} \mathrm{C}\right)$ se observa un comportamiento similar, con valores que decrecen de un 3,2\% inicial hasta 2,4\% en tercer día de almacenamiento, a partir de este momento se produce un ligero incremento de 2,6\% al cuarto día de almacenamiento. (Figura 1). 


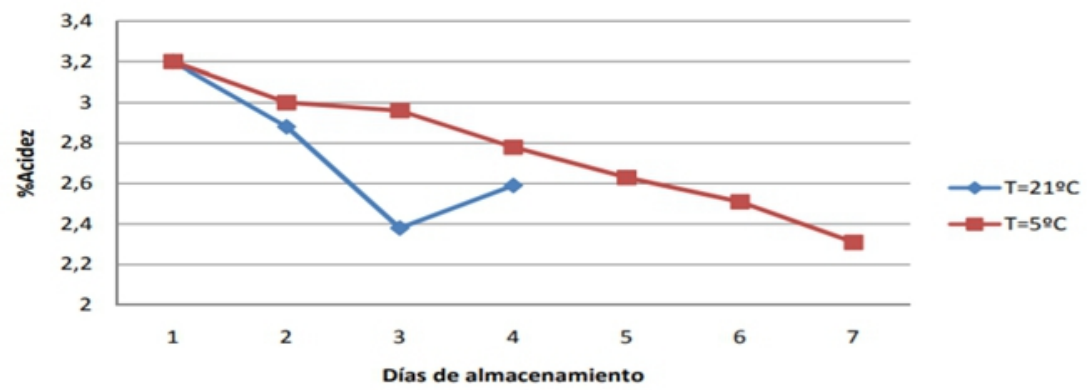

Figura 1. Variación del porcentaje de acidez titulable de la mora de castilla almacenada a dos temperaturas

La disminución presentada en los valores de acidez titulable medida en mora de castilla, responde a que de manera general los ácidos del fruto se usan como substrato de respiración, ya que los ácidos en comparación con los carbohidratos, contienen más oxígeno como parte de su estructura molecular, determinando de esta forma una liberación mayor de $\mathrm{CO}_{2}$.

Hay que anotar también que durante el almacenamiento a temperatura ambiente $\left(21^{\circ}\right)$ estos cambios ocurren con una tasa de incidencia mayor, lo que quiere decir que esta fruta presenta gran susceptibilidad a ser almacenada a temperaturas mayores de $8^{\circ} \mathrm{C}$, lo que deriva en la pérdida de calidad y disminución de la vida útil del producto.

En cuanto a la determinación de $\mathrm{pH}$, se plantearon de igual manera dos condiciones de almacenamiento a $21^{\circ} \mathrm{C}$ y $5^{\circ} \mathrm{C}$. En las dos condiciones propuestas se puede evidenciar un comportamiento ligeramente ascendente (Figura 2). En la temperatura de $21^{\circ} \mathrm{C} \mathrm{el} \mathrm{pH}$ de la mora varió de 2,98 a 3,18 valor obtenido al cuarto día de almacenamiento; y bajo refrigeración varió de 2,98 el día de la cosecha a 3,15 al sexto día, luego del cual descendió hasta un valor de 3,13 al séptimo día de almacenamiento.

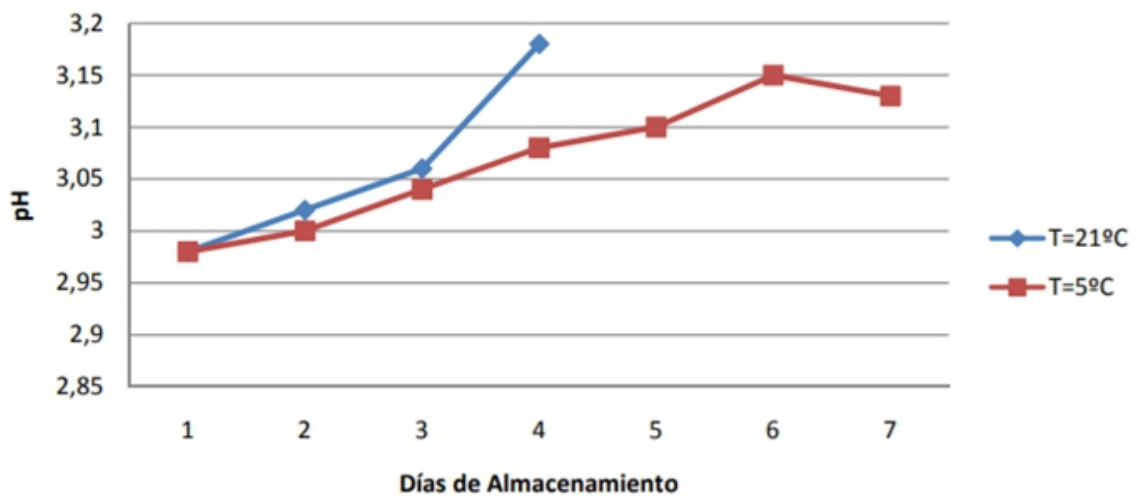

Figura 2. Variación del pH de la mora de castilla almacenada a dos temperaturas 
El valor del $\mathrm{pH}$ es usado como base para medir la madurez de las frutas por lo que es lógico establecer una relación directa con el porcentaje de acidez. La mora, objeto de nuestro estudio, al ir sufriendo un proceso normal de maduración postcosecha, presenta cambios fisiológicos normales que van a alterar sus características fisicoquímicas disminuyendo su porcentaje de acidez e incrementando progresivamente su valor de $\mathrm{pH}$. Con estos cambios de igual manera se presentan alteraciones organolépticas a lo largo del almacenamiento manifestada en modificaciones de color y apariencia.

Se realizó el recuento microbiano de mohos y levaduras efectuándose siembras periódicas a partir de los días: cero, uno, cuatro, siete y doce, constituyéndose este estudio en una de las respuestas experimentales de nuestro trabajo. Luego del análisis se presenta un valor inicial de $17 \times 103$ $\mathrm{UFC} / \mathrm{g}$. En las condiciones de almacenamiento de temperatura ambiente existe un incremento mayor de carga microbiana. Figura 3.

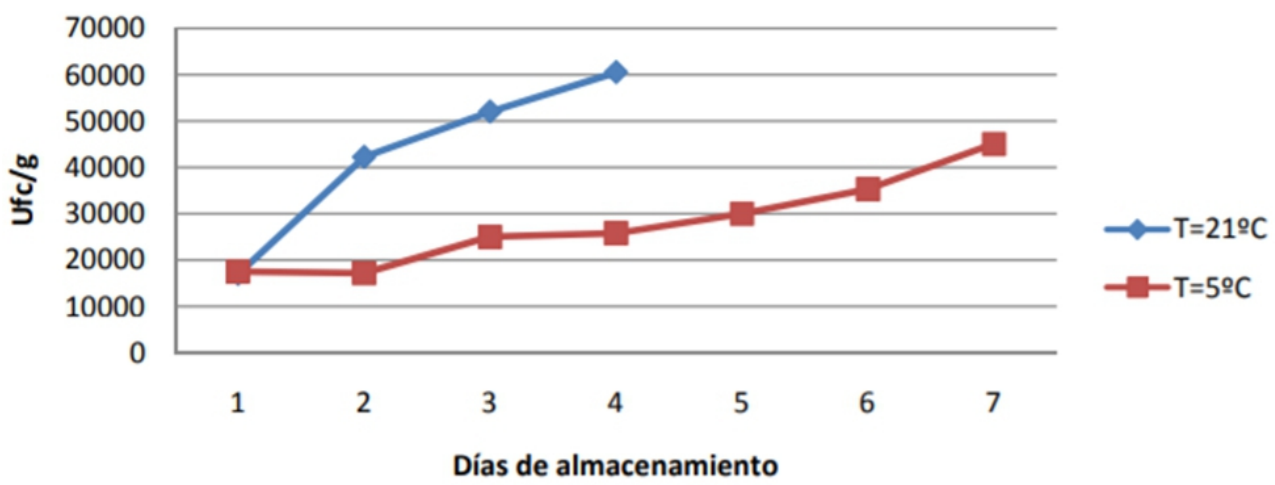

Figura 3. Variación crecimiento de mohos y levaduras mora de castilla almacenada a dos temperaturas

Aunque no exista una relación estadística entre recuento de mohos y levaduras con variables físico químicas y organolépticas como la acidez y el $\mathrm{pH}$, se pueden establecer asociaciones con respecto a los resultados obtenidos. En el caso de $\mathrm{pH}$ y porcentaje de acidez, se ven influenciados por la presencia de hongos en el fruto, ya que las transformaciones de los azúcares en ácidos orgánicos por las actividades metabólicas de los mismos, hacen que existan variaciones propias del proceso de fermentación. Hay que considerar que el incremento de la población de hongos está estrechamente relacionado con la temperatura de almacenamiento, por esta razón se presentan cambios más drásticos en temperaturas de almacenamiento de $21^{\circ} \mathrm{C}$ en comparación con las temperaturas de refrigeración $\left(5^{\circ} \mathrm{C}\right)$.

2. Determinación de los hongos causantes de la pudrición de mora de castilla

De los análisis de material vegetal (mora fresca) con síntomas de pudrición realizados, se obtuvieron aislamientos de Botrytis sp, además de 
encontrarse la presencia de Verticillium sp. Luego del análisis de los aislamientos seleccionados mediante las claves taxonómicas se determinaron características morfológicas y de crecimiento de Botrytis sp. Las características del micelio observadas fueron: hifas y conidios translucidos de color claro, redondeados de micelio hialino septado se diferencian conidióforos hialinos, delgados, lisos y ramificados irregularmente en la parte superior. Se observaron conidias hialinas, unicelulares, lisas, de formas ovoides, subesféricas a esféricas.

Este género incluye muchas especies patógenas de plantas (Ellis, 1971). Sería conveniente la aplicación de técnicas moleculares para facilitar la identificación a nivel de especie. En los cultivos de Botrytis $s p$ en su fase inicial se presentaron características macroscópicas claras como colonias algodonosas de color blanco que al transcurrir del tiempo cambiaron a una coloración café obscuro, en esta etapa de desarrollo se observaron la presencia de esporas (conidios), y posteriormente esclerocios. (Figura 4). En el caso de Botrytis sp. En agar de esporulación, presenta como una colonia amarillo pálido en el anverso y el reverso de color amarillo al centro con un borde amarillo rojizo y con textura granulosa.
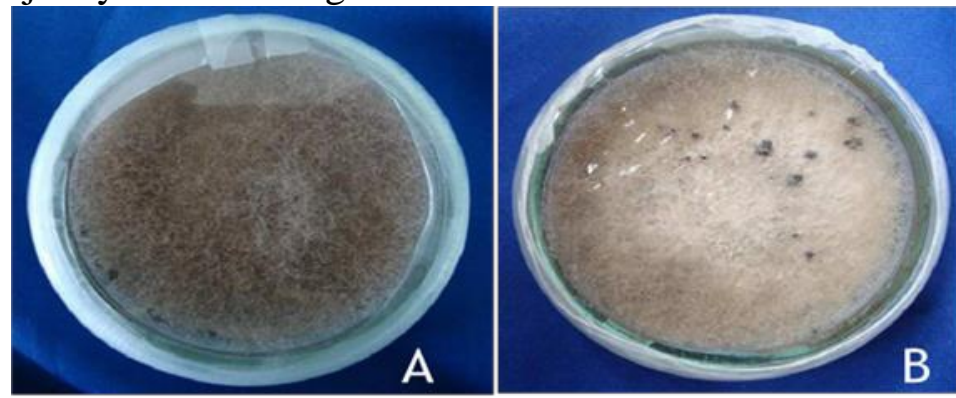

Figura 4. Morfología de la colonia de Botrytis sp. En (a) micelio, (b) micelio con esclerocios.

\section{Actividad antifúngica del aceite esencial de canela in vitro.}

El aceite esencial de canela presentó un efecto antifúngico sobre Botritys sp. (Tabla 2). A partir de la dosis mínima probada, $125 \mathrm{ppm}$, se presentó una inhibición del desarrollo del hongo. A partir de una concentración de $250 \mathrm{ppm}$ del aceite esencial, se inhibió completamente el crecimiento de la colonia de Botritys sp. El crecimiento micelial en el aceite de canela puro fue nulo, debido a una mayor cantidad e interacción de los compuestos volátiles. 
Tabla 2. Efecto del aceite esencial de canela sobre crecimiento micelial (mm) "in vitro" de Botrytis sp.

\begin{tabular}{ccc}
\hline $\begin{array}{c}\text { Aceite esencial } \\
\text { (ppm) }\end{array}$ & $\begin{array}{c}\text { Crecimiento de la } \\
\text { colonia (cm) }\end{array}$ & $\begin{array}{c}\text { Grado de } \\
\text { inhibición (\%) }\end{array}$ \\
\hline Canela & & \\
$\mathbf{0}$ & $8.1 \mathrm{a}^{*}$ & 83 \\
$\mathbf{1 2 5}$ & $1.4 \mathrm{~b}$ & 100 \\
$\mathbf{2 5 0}$ & $0 \mathrm{c}$ & 100 \\
$\mathbf{5 0 0}$ & $0 \mathrm{c}$ & 100 \\
$\mathbf{1 0 0 0}$ & 0c & 100 \\
\hline
\end{tabular}

*Letras diferentes en la columna indican diferencias significativas (Tukey, $\mathrm{p}<0.05$ ).

Como se pudo observar, los cambios químicos que ocurren en la mora de castilla bajo almacenamiento en los días postcosecha se encuentran en un rango relativamente pequeño. De ahí la importancia del grado de madurez con que se coseche este producto. La disminución de los ácidos en el fruto, indica, generalmente, que se están utilizando, forzadamente, como substrato de respiración (Seymour et al., 1993), debido a que los ácidos, en comparación con los carbohidratos, contienen, por cada átomo de $\mathrm{C}$ y de $\mathrm{H}$, más átomos de O y así, la liberación de CO2es mayor que la toma de O2 (Schultz, 1993).

En el reglamento sanitario de los alimentos está especificada la categoría de los productos hortícolas mínimamente procesados siendo el límite para el recuento de mohos y levaduras de 105UFC/g (López 1999), observando que el recuento de mohos y levaduras luego del análisis se presenta un valor inicial de $17 \times 103 \mathrm{UFC} / \mathrm{g}$. En las condiciones de almacenamiento de temperatura ambiente existe un incremento mayor de carga microbiana. Figura 3. Hay que anotar que a mayor temperatura de almacenamiento estos cambios ocurren a una tasa mucho más alta; lo anterior quiere decir que esta fruta tiene gran susceptibilidad a ser almacenada a altas temperaturas (mayores de $8^{\circ} \mathrm{C}$ ), en lo que se refiere a la duración de la vida útil del producto.

La alta capacidad antifúngica encontrada en el aceite esencial de $C$. zeylanicum coincide con los resultados reportados por Montes y Carvajal (1998) quienes observaron que Aspergillus flavus fue totalmente inhibido por el aceite esencial de esta planta. Wilson et al. (1997) evaluaron 49 aceites esenciales y encontraron que $C$. zeylanicum mostró la mayor actividad antifúngica contra Botrytis cinerea. Estos autores también publican la composición química de los aceites esenciales y la correlación entre la concentración de los compuestos y la actividad antifúngica.

De acuerdo con los resultados, se observó que a 125 ppm se presentó el mayor crecimiento micelial del hongo con respecto a las otras 
concentraciones. Mientras que a partir $250 \mathrm{ppm}$ no hubo crecimiento micelial (Figura 5). También se observó que conforme aumentó la concentración del aceite esencial, el efecto de inhibición sobre el crecimiento micelial es mayor, esto significa que se presentó un efecto dependiente de la concentración. Esto coincide con los resultados obtenidos por Barrera- Necha et al., (2008) que utilizaron aceites esenciales de clavo, canela y tomillo a concentraciones de $100,150,200,250$ y $300 \mu \mathrm{g} \mathrm{mL}-1$ sobre C. gloeosporioides, observando que conforme aumentó la concentración de los aceites esenciales la inhibición del crecimiento micelial fue mayor. García, E. et al. (2006), observaron que existe una relación entre el incremento de la concentración del Aceite esencial y la inhibición en la producción de aflatoxinas por A. flavus, en nuez pecanera. Con los resultados de este ensayo se decidió utilizar solo las concentraciones de 250 y 500 ppm.
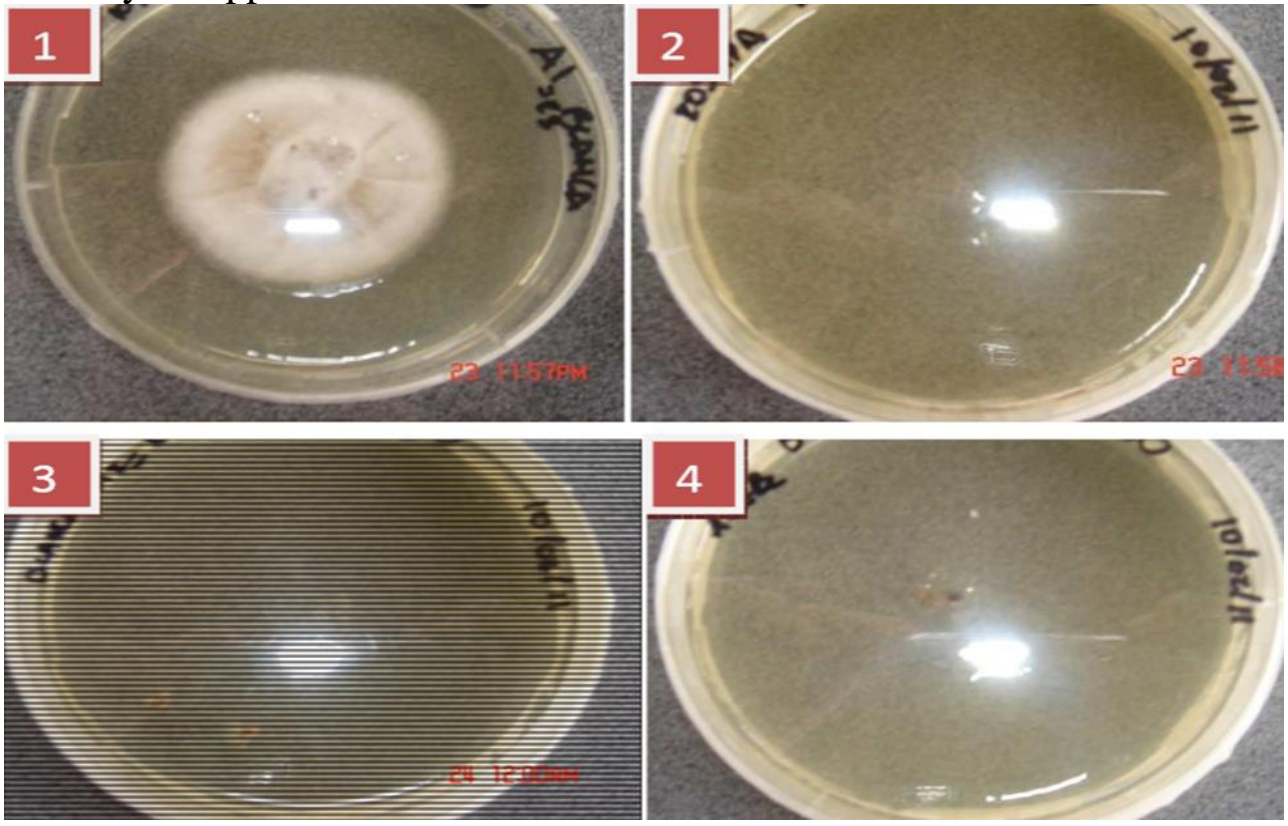

Figura 5. Crecimiento micelial de Botrytis sp después de 7 días de la aplicación del aceite esencial a cuatro concentraciones $125 \mathrm{ppm}(1), 250 \mathrm{ppm}(2), 500 \mathrm{ppm}$ (3) y 1000ppm(4)

Estos resultados coinciden con lo reportado por Soliman y Badeaa (2002) quienes encontraron que la canela inhibió completamente el desarrollo de Botritys sp. en una dosis de $500 \mathrm{ppm}$. Los tres componentes del aceite de canela que han sido identificados como los agentes activos contra hongos son: aldehído cinámico (Bullerman, 1977), Ometoxicinamaldehído (Morozumi, 1978) y eugenol (Velluti et al., 2003). La dosis mínima fungicida para el aceite esencial de canela fue de $1000 \mathrm{ppm}$ para el aceite esencial de canela. 


\section{Conclusiones}

El presente estudio permitió comparar la actividad antimicrobiana del aceite esencial de canela mediante el método de microdilución. El aceite esencial de canela (Cinnamomum zeylanicum), presentó un efecto antifúngico sobre Botritys sp. A partir de la dosis mínima probada, 125 ppm, se presentó una inhibición del desarrollo del hongo. A partir de una concentración de 250 ppm del aceite esencial, se inhibió completamente el crecimiento de la colonia de Botrytis sp, por lo que este aceite es una alternativa atractiva para el control de enfermedades causadas por Botrytis en fruta fresca.

Las concentraciones mínimas inhibitorias y fungicidas fueron: $250 \mathrm{y}$ 500 ppm (títulos de las soluciones testadas) inhibiendo el crecimiento micelial del Botrytis sp. La aplicación del aceite esencial de Canela en concentraciones de 250 y 500 ppm retardó significativamente el aumento de $\mathrm{pH}$ a lo largo del período de almacenamiento a temperatura ambiente, así como retardó el crecimiento de mohos y levaduras presentando diferencias significativas con respecto al crecimiento de mohos y levaduras de la fruta sin recubrir.

\section{References:}

1. Ayala, S. Valenzuela, R. Bohórquez, P. (2013): "Variables determinantes de la madurez comercial en la mora de Castilla (Rubus glaucus Benth)", Revista Scientia Agroalimentaria, 1: 39-44, 2013, ISSN:

4684, Disponible en: http://revistas.ut.edu.co/index.php/scientiaagro/ article/view/29

2. A.O.A.C. (2000). Oficial. Methods of Analysis. (16 ed). Washington. D.C. pp. $376-384$.

3. Adams M.R., Moss, M.O. (1997). Microbiología de los Alimentos. Zaragoza: Editorial Acribia, 1997. 176 p.

4. Akgul, A., Kivanc M. (2006). Inhibitory Effect of Six Turkish Thymelike Spices on Some Common Food-Borne Bacteria. Molecular Nutrition \& Food. Volumen (32). 201-203.

5. Ardila N., Luis R., (2001, 3 de enero). Producción de mora de castilla. Recuperado de: http://www.agriculturasensitiva.com/mora.htm

6. Barrera, L., Garduno, C., \& García, L. (2009). In vitro Antifungal Activity of Essential Oils and Their Compounds on Mycelial Growth of Fusarium oxysporum f. sp. gladioli (Massey) Snyder and Hansen. 8, 17-21. doi:10.3923/ppj.2009.17.21

7. Barrera-Necha, L. L., Bautista-Baños, S., Flores-Moctezuma, H. I. y Rojas-Estudillo, A. (2008). Efficacy of essential oils on the conidial germination, growth of Colletotrichum gloeosporioides(penz.) Penz. And Sacc. and control of postharvest diseases in papaya (Carica papaya L.). Plant Pathology Journal 7 (2): 174-178. 
8. Bejarano, W. (1992) Manual de Mora (Rubus glaucus Benth). Quito Ecuador, Proexant. pp. 6-12; 42-60.

9. Bullerman, L.B., Y. Lieu and S.A. Seier. (1977). Inhibition of growth and aflatoxin production by cinnamon and clove oils, cinnamic aldehyde and eugenol. J. Food. Sci. 42:1107-1109.

10. Carmona, M.J.; et al. (2006) Caracterización Fisicoquímica de Seis Materiales de Mora (Rubus glaucus Benth). Agronomía Colombiana, julio/diciembre, Volumen (24). 306-316.

11. Ellis, MB (1971) Dematiaceous Hyphomycetes. CMI. Kew Surrey FAOSTAT (2014) Beans harvested area and production. Recuperado de: http://www.fao.org/statistics.

12. Fernandez X. (2010, 25 de agosto). El cultivo de mora en Ecuador. SICA. Recuperado de: http://www.sica.gov.ec/agronegocios/biblioteca/Ing\%20Rizzo/nuevos $\%$ 20expo rtables/mora/cultivo.htm.

13. FAO. 2006. Cinnamomum oils (including cinnamon and cassia). Forestry Dept. Disponible en: http://www.fao.org/docrep/V5350E/V5350e04.htm.

14. Galeana, S., Morales V. (2002). Preconservación de Durazno por Métodos Combinados: rehúso del jarabe y estabilidad de la fruta de empaque. (Tesis de pregrado). Universidad de las Américas, Puebla.

15. Galvis S.J., Herrera A. (2005). Manejo Postcosecha de Mora. Bogotá: Servicio Nacional de Aprendizaje (SENA)..

16. García, E., Quezada , Y., Moreno, J., Sánchez, G., Moreno , E., \& Pérez, M. (2006). Actividad Antifúngica de Aceites Esenciales de Canela (Cinnamomum zeylanicum Blume) y Orégano (Origanum vulgare L.) y su Efecto sobre la Producción de Aflatoxinas en Nuez Pecanera (Carya illinoensis (F.A. Wangenh) K. Koch). Redalyc, 24(1). Obtenido de https://www.redalyc.org/html/612/61224102/

17. Guzmán, T. Cuenca, K. Tacuri, E.: Caracterización de la poscosecha de la mora de Castilla (Rubus glaucus) tratada con 1 metilciclopropeno, Volumen (27), $\mathrm{N}^{\circ} 1$. Recuperado de: http://scielo.sld.cu/scielo.php?script=sci_arttext\&pid=S207100542018000100007

18. ICONTEC. (1997). INSTITUTO COLOMBIANO DE NORMAS TÉCNICA. Frutas Frescas: Mora de Castilla, especificaciones. NTC4106. Bogotá: ICONTEC.

19. INEN (2010). Frutas Frescas. Mora. Requisitos. NTE INEN 2427. Quito - Ecuador.

20. Jayaprakasha G.K. (2007). Antioxidant and Antimutagenic Activities of Cinnamomum zeylanicum Fruit Extracts. Journal of Food Composition and Analysis. Volumen (20). 330-336. 
21. Jiménez E., Amador F., Tijerino N. (2006, 20 de agosto). Insectos plagas y benéficos asociados al cultivo de mora (Rubus glaucus benth). Recuperado de: http://www.uca.edu.ni/publicaciones/encuentro/e75/E75T3.html

22. Kaume, L. Howard, L. Devareddy, L. KAUME, L.: “The Blackberry Fruit: A Review on Its Composition and Chemistry, Metabolism and Bioavailability, and Health Benefits", Journal of Agricultural and Food Chemistry, 60(23): 5716-5727, 2012, ISSN: 0021-8561, DOI: 10.1021/jf203318p, Disponible en: http://dx.doi.org/10.1021/jf20331 $8 \mathrm{p}$

23. Montes R, Carvajal M. (1998). Control of Aspergillus flavus in maize with plant essential oils and their components. Journal of Food Protection Vol: 61: 616-619.

24. Morozumi, S. (1978). Isolation, purification and antibiotic activity of o -methoxycinnamaldehyde from cinnamon.Applied Environmental Microbiology 36:577-583.

25. Plotto A.; D. D. Roberts and R. G. Roberts. (2003). Evaluation of plant essential oils as natural postharvest disease control of tomato (Lycopersicon esculentum). Acta Hort. 628:737-745.

26. Ramírez, J. (2012): Conservación de mora de castilla (Rubus glaucus Benth) mediante la aplicación de un recubrimiento comestible de gel de mucílago de penca de sábila (Aloe barbadensis Miller), [en línea], Universidad Nacional de Colombia, Sede Medellín, Tesis de Maestría, Medellín, Colombia, $112 \quad$ p., , Disponible en: http://www.bdigital.unal.edu.co/6911/

27. Raybaudi R., Soliva R., Martín O. (2016) Uso de agentes antimicrobianos para la conservación de frutas frescas y frescas cortadas. En: simposio iberoamericano de vegetales frescos cortados. (1: Abril, 2006. San pedro, Sao Paulo). Sao Paulo.

28. Reina C A. (1998). Manejo poscosecha y evaluación de la calidad de la mora de Castilla (Rubus glaucus) que se comercializa en la ciudad de Neiva. (Tesis de pregrado). Universidad Surcolombiana; 1998. 133 $\mathrm{p}$

29. Saltos A. (2001). Investigación y Desarrollo de Tecnologías Aplicadas a la Conservación de Frutas - Mora de Castilla (Rubus glaucus Benth). Ambato - Ecuador: FUNDACYT. 74-94.

30. Sanchez. L. et al. (2008). Incorporación de productos naturales en recubrimientos comestibles para la conservación de alimentos. En: Congreso SEAE (8: 16-20, Septiembre: Bullas, Murcia). Bullas.

31. Seymour, G., J. Taylor y G. Tucker (eds.). (1993). Biochemistry of fruit ripening. Chapman and Hall, London. 
32. Soliman K.M. and R. I. Badeaa. (2002). Effect of oil extracted from some medicinal plants on different mycotoxigenic fungi. Food Chem. Toxicol. 40(11):1669-1675.

33. Schultz R. (1993). Proteínas fisiológicas. En: Devlin T (ed.). Bioquímica. Barcelona: Reverté; p. 95-133.

34. Tsao. Zhou. (2000) Antifungal Activity of Monoterpenoids Against Postharvest Pathogens Botrytis cinerea and Monilinia fructicola. Journal Essential Oil Research. Volumen (12). 113-121

35. Villegas, C. Albarracín, W. Osorio, O. (2016): "Blackberry (Rubus glaucus Benth) preservation by edible coating application", Vitae, 23: 727-730, ISSN: 2145-2660.

36. Velluti A.; V. Sanchis, A. J. Ramos, J. Egido and S. Marin. (2003). Inhibitory effect of cinnamon, clove, lemongrass, oregano and palmarose essential oils on growth and fumonisin B1 production by Fusarium proliferatum in maize grain. Int. J. Food Microbiol. 89(23):145-154.

37. Wilson, C. L.; J. M. Solar, A. El Ghaouth and M. E. Wisniewski. (1997). Rapid evaluation of plant extracts and essential oils for antifungal activity against Botrytis cinerea. Plant Disease 81:204-210. 